\title{
"We take the risk of hope" Überlegungen zu akademischer (Reproduktions-)Arbeit im Anschluss an das Vernetzungstreffen „Feministische Geographien“ in Hamburg
}

\author{
Nina Fraeser, Sarah Klosterkamp, \\ Juliane Kühn, Eva Kuschinski \\ und Theresa Martens
}

Die Beweggründe für das kollektive Verfassen dieses Artikels sind die systematischen Widersprüche zwischen effizienzorientierter universitärer Arbeit und Geniemythos der Wissenschaft einerseits und den Ansprüchen an kritische - und feministische - Lehrpraxis und widerständige Alltagspraxen in der Akademie andererseits. Unsere Motivation basiert also sowohl auf der Sichtbarmachung von Missständen, als auch auf Freude, Leidenschaft und Kollegialität. Anschließend an Diskussionen zu Bedingungen kritischer Wissensproduktion (bspw. s u b $\backslash \mathrm{u}$ r b a n 2016, Band 4, Heft 2/3) argumentieren wir, dass ein Blickwinkel auf akademische Arbeit als Care- und Reproduktionsarbeit unabdingbar ist und gleichzeitig emanzipatorische Ansätze zum Lehren, Lernen und Forschen (entsprechend einer feministischen Praxis) ermöglicht.

Zunächst wird geschildert, wie Verantwortungsabgabe dazu beiträgt, sich vom Vorbereitungs- und Erfolgsdruck akademischer Tagungen zu distanzieren und Raum zu schaffen für ergebnisoffene und solidarische wissenschaftliche Arbeitsweisen. Des Weiteren wird die Verschränkung von kritischer Wissensproduktion und feministischer Praxis beleuchtet und somit die Universität als Ort der Reproduktion konzeptualisiert. Abschließend verknüpfen wir unsere Überlegungen zu Stadt und Reproduktionsarbeit mit gegenwärtigen politischen Praxen der solidarischen und kollektiven Alltagsbewältigung und zeigen damit auf, wovon wir in der Akademie lernen können.

Maßgeblich inspiriert ist dieser Text durch das Vernetzungstreffen „Feministische Geographien“[1], das Ende April 2017 in Hamburg stattgefunden hat. Die Autorinnen schreiben diesen Text daher in einem situativen, solidarischen Wir. Situativ, weil sich dieses Wir zu unterschiedlichen Momenten unseres Erzählens verschieden zusammensetzt. Mal schreiben wir als Teil des OrgaTeams des Treffens, mal als Teilnehmerinnen, dann wieder als Beschäftigte der Akademie oder Feministinnen. Solidarisch ist unser Wir, weil wir in der Unterschiedlichkeit unserer Positionen, ob in Bezug auf die Universität[2] oder unsere Feminismen, aufgrund unserer gemeinsamen Erfahrungen während des Treffens in Hamburg und darüber hinaus eine solidarische Praxis erproben und uns darin verbunden fühlen. Hierzu gehört auch das Verfassen dieses 
Textes. Unsere Stimmen sind verwoben mit denen von Studierenden und Kolleg_innen, wir schöpfen aus Gesprächen während und nach dem Treffen.[3] Wir wollen Erfahrungen und Überlegungen teilen und zur Diskussion stellen. Sie sind aus dem Bewusstwerden der Differenz zwischen den Arbeitsweisen während des Vernetzungstreffens und dem universitären Alltag, zu dem die meisten von uns danach zurückgekehrt sind, entstanden.

Insgesamt haben circa 50 Personen aus unterschiedlichen Universitätsstandorten Deutschlands am Vernetzungstreffen „Feministische Geographien“ teilgenommen. Die meisten davon sind Geographie-Studierende, aber auch Beschäftigte im Wissenschaftsbetrieb, niemand davon mit Professur. Das Orga-Team aus Hamburg hatte im Vorfeld nur eine inhaltliche Setzung vorgenommen: die aktuellen Krisentendenzen und Verschiebungen im Bereich der Reproduktionsarbeit. Vor allem haben wir jedoch, um Zeit und Raum für die Entwicklung gemeinsamer Themen zu schaffen, die BarCamp-Methode[4] für die Strukturierung des Treffens genutzt und damit folgende temporären Arbeitsgruppen gebildet: Reflexivität in der (Feld-)Forschung (daraus hervorgegangen: Mürlebach et al. 2017), feministische Perspektiven auf akademische Arbeit, feministisch-psychoanalytische Forschungsansätze, postkoloniale Stadtforschung und indigener Feminismus in Lateinamerika. Somit konnten wir uns mit Inhalten auseinandersetzen, die in unserem Studien- und Arbeitsalltag oft zu kurz kommen, auch weil sie feministisch sind.

\section{„Ich soll eine Abendveranstaltung moderieren?“ - solidarische Aufgabenverteilung}

„[...W] take the risk of hope with full knowledge of the possibility, even the certainty, of failure."[5]

Der entscheidende Knackpunkt besteht für uns darin, entgegen einer professionalisierten und erfolgsorientierten Selbstausbeutung, die oft mit der Organisation wissenschaftlicher Konferenzen einhergeht, die logische Konsequenz zu ziehen, sich von Planbarkeit zu lösen und der Möglichkeit des Scheiterns erwartungsvoll gegenüberzustehen. Der universitäre Alltag lässt oft nur wenig zusätzliche Energie und Zeit für extracurriculare Aufgaben wie die Organisation eines Vernetzungstreffens. Es gab Momente, in denen einem das vormals ganz optimistisch in der Ferne schillernde Event plötzlich wie ein Klotz vor die Füße fiel: Der schlaue Einfall zum inhaltlichen Workshop kam immer noch nicht und das Gefühl, sicher etwas Wichtiges vergessen zu haben, schlich sich langsam ein. Zudem waren wir uns als Organisierende unsicher darüber, ob unser Konzept für das Treffen so aufgehen würde, wie erhofft. Denn dieses bestand auch darin, viel Kontrolle abzugeben und sich auf das unbekannte Kollektiv der Teilnehmenden zu verlassen.

Dabei ging es zunächst um die Verteilung klassischer Reproduktionsarbeit, wie zum Beispiel der Unterstützung der (externen) Kochgruppe. Wenn es aber um die Übernahme sowie Abgabe anderer Aufgaben ging - wie die inhaltliche Moderation einer öffentlichen Diskussionsveranstaltung -, kostete es uns weitaus mehr Überwindung. Eine weitere Konsequenz unserer aktiven Abwendung von etablierten akademischen Tagungsstrukturen war das Ausprobieren anderer Formate des Lernens, jenseits von Performance-, 
Noten- und Zeitdruck. So haben wir beispielsweise von einem klassischen Keynote-Vortrag abgesehen, weil wir selbst oft genug erfahren hatten, dass man selten Zeit für die vorbereitende Lektüre findet oder sich nicht traut, in einem solchen Setting voller vermeintlich versierterer Personen etwas zu sagen. Die Vorbereitung auf die Diskussionsveranstaltung mit Julia Dück und Sarah Speck fand vor Ort statt. In Kleingruppen näherten wir uns ihrer Arbeit mit der gemeinsamen Lektüre kurzer Texte (vgl. Dück/Fried 2015, Speck o. J.) und diskutierten im Anschluss Fragen zu geographischen Perspektiven auf Reproduktionsarbeit. Es war die erste inhaltliche Auseinandersetzung während des Treffens, die neben der kollektiven Vorbereitung ermöglichte, einander besser kennenzulernen. Dabei wurden Teilnehmende dazu ermutigt Aufgaben wie das Repräsentieren einer Kleingruppe während der Diskussionsveranstaltung oder auch die Moderation eben dieses Formates zu übernehmen, die bisher kaum Erfahrung mit derlei Tätigkeiten hatten.

Somit wurde die klassische Unterteilung zwischen den unsichtbaren,fleißigen Händen 'im Hintergrund und den routinierten Expert_innen, die häufig ausschließlich für die Inhalte solcher Treffen zuständig sind, aufgebrochen. Wir möchten nicht behaupten, dass wir damit Hierarchien oder gesellschaftliche Machtverhältnisse aufgelöst haben. Dennoch bleibt für uns nach dem Treffen das Gefühl, dass wir es geschafft haben, an diesem Wochenende durch Anerkennung und Wertschätzung der unterschiedlichen Wissens- und Erfahrungsbestände der Teilnehmenden, ähnlich wie es Bürk (2016: 236) vorschlägt, eine ergebnisoffene und solidarische wissenschaftliche Praxis zu erproben, die sich unterscheidet von den etablierten Strukturen unserer Arbeits- und Studienrealitäten. Auf dieser Erfahrung können wir aufbauen, denn sie gibt Kraft für die Auseinandersetzung mit Professionalisierungsund Leistungszwang im Hochschulalltag.

\section{Über das akademische Viertel hinaus - Wissensproduktion ohne Zeit- und Bewertungsdruck}

Ein Versuch, sich genau von diesen Zwängen ein Stück weit freizumachen, stellte das Format des BarCamps dar, aus dem sich eine Vielfalt an Themen ergab. Nach der Präsentation dieser Methode stand der Großteil von uns mehrere Minuten im Raum und wägte ab, für welches Thema die gemeinsame Zeit in den jeweiligen Arbeitsgruppen genutzt werden sollte. Es tat gut, die Entscheidung nicht entlang der Erfüllung von Prüfungsleistungen oder Antragsgeldern treffen zu müssen; sich Zeit zu nehmen für eine solche Arbeitsweise empfanden wir als Luxus jenseits alltäglicher Effizienz- und Strategiegedanken.

Neben einer kritischen Wissensproduktion, welche gesellschaftliche Verhältnisse in Frage stellt, glauben wir, dass es einer Praxis bedarf, in der wir mehr füreinander sorgen, um den prekären Verhältnissen im Universitätsalltag zu begegnen. Auf dem Treffen stellten unsere prozessorientierte Arbeitsweise und die flexibleZeiteinteilung innerhalb der Arbeitsgruppen eine Bereicherung für uns dar. Wir konnten innerhalb offener Strukturen diskutieren und über die Zeit selbstbestimmt verfügen, um zwischen einzelnen Arbeitsphasen einen power-nap einzulegen oder spazieren zu gehen. In unserem eigenen Rhythmus 
zu denken und auch die Selbstsorge nicht außer Acht zu lassen, ist für uns ein essentieller Bestandteil feministischer Wissensproduktion.

In der Arbeitsgruppe zum Thema „Feministische Perspektiven auf akademische Arbeit" formulierten einige Studierende, die in Bonn autonom Seminare zu feministischen Geographien organisieren, ein sehr deutliches Bewusstsein darüber, dass ihre Studienbedingungen im direkten Zusammenhang mit den Beschäftigungsverhältnissen an den Universitäten stehen. Für diejenigen unter uns, die als Dozentinnen davon betroffen sind, war das ein besonders wichtiger Moment. Wir haben uns nicht nur über gemeinsam gelesene Texte, sondern auch über Erfahrungen aus dem wissenschaftlichen beziehungsweise universitären Alltag und die unentlohnte Arbeit, die wir jeweils in diesem Kontext leisten, ausgetauscht. So konnten wir erkennen, dass sowohl Studierende als auch Dozierende jeweils Expert_innen mit wichtigen Perspektiven sind. Durch den kontinuierlichen Austausch auf Augenhöhe ließen sich Allianzen schmieden, in denen wir gemeinsam den zunehmenden Neoliberalisierungsprozessen an den Universitäten und Forschungsinstituten entgegentreten können (vgl. Mountz et al. 2015, Bürk 2016).

Aus dieser gelebten feministischen Praxis ergab sich neben der guten Stimmung und der intensiven, solidarischen Vernetzung auch eine Befruchtung der inhaltlichen Diskussion. Als wir mit Julia Dück die Thesen diskutierten, dass Care-Arbeit nicht ohne massiven Qualitätsverlust in ihrer Effizienz steigerbar ist und dass die Arbeitsverhältnisse des Pflegepersonals an der Charité und das Wohl der Patient_innen direkt zusammenhängen, konnten wir Parallelen zu unserem Austausch ziehen. Wir haben somit eine neue Perspektive auf die Universität als Ort der Reproduktion gewonnen. Zumindest insofern, als dass auch akademische Arbeit nicht in der Effizienz steigerbar ist, ohne dafür bedeutend an Qualität zu verlieren, sodass auch der Bildungsbetrieb Universität als Ort der Reproduktion gelesen und bewertet werden sollte.

Zunächst einmal ist von denjenigen, die in der Lehre arbeiten, CareArbeit in der Betreuung von Studierenden zu leisten. Wir setzen uns oft mit Problemen von Studierenden auseinander, wenn es um verspätete Abgaben oder nicht erbrachte Leistungen und deren Begründung geht. In Momenten, in denen aus der Position der Studierenden das Bedürfnis besteht, als Person erkannt zu werden, versuchen wir als Dozentinnen empathisch und solidarisch, Vergleichbarkeit und Fairness suggerierende Mechanismen wie Abgabetermine biegbar zu machen. Gleichzeitig sind lebendig beschriebene Überforderungen oder Schicksalsschläge zwar selten Probleme, zu deren Lösung wir beitragen können, jedoch begleiten sie uns oft weiter durch unseren (All-)Tag: In einer Care-Kette sprechen wir dann mit Kolleg_innen über die Hürden der Studierenden und die eigenen Schwierigkeiten damit umzugehen. Gemeinsam finden wir Lösungen und wägen ab, welche Möglichkeiten wir innerhalb oftmals stark reglementierter Verwaltungsstrukturen haben. Da kommt es auch vor, dass ein Konferenz-Abstract nicht geschrieben wird oder Texte für die nächste Seminarvorbereitung nur überflogen werden. Als feministische Praxis ist diese Art von Care jedoch ein Anspruch, den viele von uns an sich selbst stellen.

Aus der Perspektive vieler Studierender wurde in Gesprächen während des Treffens und danach immer wieder darauf verwiesen, dass in einer neoliberalisierten Universität Konkurrenz und Hierarchien schon durch 
Benotungen stets präsent sind. Die Bologna-Reform hat ihren Teil dazu beigetragen, dass Noten bereits in den ersten Jahren des Studiums als wichtig erachtet werden. Aber auch in der Qualität von Lehrveranstaltungen schlagen sich diese Prozesse nieder. So greifen prekär beschäftigte Dozierende häufig auf Lehrformate zurück, die zu großen Teilen aus Referaten der Studierenden bestehen und somit nur begrenzt Raum für kollektive Lernprozesse bieten. Aus der Perspektive einer - wenn auch temporären - solidarischen Praxis wurden für uns die Möglichkeiten, Verschränkungen und Herausforderungen der unterschiedlichen akademischen Statusgruppen und ihrer Kämpfe um Reproduktionsverhältnisse des universitären Alltags deutlich.

Wir konnten neue, wertvolle Perspektiven auf unsere Arbeit gewinnen und somit einen gemeinsamen und solidarischen Wissensbestand schaffen. Dies ermöglichte uns auch, der - von Schuster (2016: 194f.) in Bezug auf Dölling (2013) proklamierten - etablierten Hierarchisierung von akademischem und Bewegungs-Feminismus eine andere Praxis entgegenzusetzen. Der Austausch von Erfahrungswissen kann demnach die Basis für feministische Theoriearbeit sein. Durch die Betrachtung von universitärer Arbeit als Reproduktionsarbeit wollen wir Prozesse der Individualisierung, Unsichtbarmachung und Entwertung von Reproduktionsarbeit an der Akademie eine weitere Sprecherinnenposition entgegensetzen.

\section{Zwischen Latte Macchiato und Care-Streik - Von (feministischen) Alltagskämpfen in der Stadt lernen?!}

Während des Treffens widmeten wir uns mehrfach der Stadt als Ort, der vielfältige Perspektiven einer feministischen Analyse von Reproduktionsarbeit zulässt, aber auch lokaler Ansatzpunkt kollektiver Kämpfe um eine reelle Transformation dieser Verhältnisse sein kann. Basierend auf den Beiträgen von Sarah Speck, zu Kontinuitäten in der geschlechtsspezifischen Hausarbeit bei heterosexuellen Paaren, und Julia Dück, zum Care-Streik in der Charité, haben wir in unserer Diskussion nach urbanen Bezügen zu ihren Thesen gesucht. Im Hinblick auf den Zugang zu öffentlichen Räumen in der Stadt, wer sie nutzen darf und soll und wie dadurch Geschlechterverhältnisse sowohl verändert als auch verfestigt werden, wurde das Beispiel sogenannter ,Macchiato-Mütter (Maier 2011) besprochen. Mit diesem Begriff werden idealtypisch junge, weiße, potenziell akademisch gebildete Cis-Frauen betitelt, deren Anwesenheit im Stadtraum als aggressive Zurschaustellung des Mutterseins wahrgenommen wird. In einigen Berliner Cafés wurden daraufhin Kinderwagenverbote ausgesprochen. Hierbei wird der Kampf um Stadtteile und gegen die Veränderung ihrer Einwohner_innenstruktur deutlich. Ebenso hat der Diskurs um diese Mütter aber auch eindeutig misogyne Züge. Er zeigt, dass Frauen, die sich mit großem Selbstbewusstsein den öffentlichen Raum aneignen und öffentlich Reproduktionsarbeit nachgehen, abgewertet werden. Dieses Beispiel verdeutlicht, dass es notwendig ist, politische Kämpfe gegen die negativen Folgen von Gentrifizierung und Geschlechterpolitiken, in diesem Falle die Bedingungen von Reproduktionsarbeit, zusammen zu denken.

Um jedoch nicht nur auf der Analyseebene zu verbleiben, tauschten wir uns auch über das Potenzial von Städten als Räume der Begegnung und des Widerstands in der Krise der Care-Arbeit aus. Unter diesem Begriff der 
Krise wird der allmähliche Zusammenbruch von Versorgungseinrichtungen wie Krankenhäusern verstanden, in denen Fürsorge aufgrund von Austeritätspolitiken immer stärker gemäß den Paradigmen der Marktförmigkeit und Effizienz organisiert wird. Die staatliche Daseinsvorsorge bricht an diesen Orten zusammen und/oder weicht unternehmerischen Modellen, wodurch immer mehr Menschen der angemessene Zugang zu reproduktiven Ressourcen versperrt wird. Als Reaktion darauf wird in einer wachsenden Zahl von Städten unter dem Label der Basisorganisierung (vgl. Maruschke 2014, Kratzsch/Maruschke 2016) versucht, im Alltag solidarisch zusammenzuarbeiten: Es werden neue Stadtteilläden, Centri Sociale und Mieter_inneninitiativen gegründet. Diese Alltagsarbeit ist feministisch, denn sie birgt die Chance, die Stadt als den Ort zu begreifen, an welchem wir jetzt ein solidarisches Zusammenleben erproben können. Unsere Diskussion der Alltagskämpfe in der Stadt ist darüber hinaus aber auch mit unserem akademischen Alltag verknüpfbar. So können feministische Praxen der solidarischen Wissensproduktion in der Akademie auch von der politischen Praxis in sozialen Bewegungen lernen (vgl. Schuster 2016).

\section{An kollektiven Versuchen können wir nicht individualisiert scheitern}

Akademische Arbeit als Reproduktionsarbeit zu lesen, die einer solidarischen Praxis bedarf, ist die zentrale Konsequenz, die wir ausgehend von der Beschäftigung mit den Kontinuitäten, Brüchen und Krisen der gesellschaftlichen Care- und Reproduktionsarbeit ziehen. Durch das Zusammendenken gesellschaftlicher Verhältnisse und alltäglicher Praxen kann feministische Analyse praktisch wirksam werden: Wir erkennen die Universität einerseits als Ort der Reproduktion von Arbeitskraft mit all den dort angelegten gesellschaftlichen Herrschaftsverhältnissen (vgl. Gutiérrez-Rodríguez et al. 2016, Bürk 2016). Sie ist andererseits ein Ort der Care-Arbeit, weil wir diese dort tatsächlich alltäglich leisten und brauchen. Folglich können wir vor allem für den Bereich der universitären Lehre eine Parallele zur derzeitigen Krise der Reproduktionsarbeit ziehen, da hier Arbeitsverhältnisse und Studienbedingungen eng aneinandergekoppelt sind. Wir erkennen in diesen alltäglichen Widersprüchen das Potential für Transformation.

Und so einleuchtend diese Erkenntnisse vielleicht für die auf- und abgeklärten Leser_innen klingen mögen, so wenig selbstverständlich ist es, dementsprechend zu handeln. Denn auch wenn eine kritische Perspektive auf unsere Studien- und Arbeitsbedingungen den Widerspruch zwischen dem bestehenden (Universitäts-)System und den Ansprüchen an solidarische und emanzipatorische Wissensproduktion aufzeigt, so fehlen häufig Alltagsstrategien dagegen anzukämpfen. Durch das Aufbrechen etablierter und hierarchisierter Positionen zeigt sich, wo Allianzen zu schmieden sind.

Das Verständnis für die Position der Anderen kann erst im Austausch auf Augenhöhe entstehen. Dozierende müssen dafür Abstand vom Mythos der professionalisierten, aber auch individualisierten Wissenschaftler_in nehmen. Ein Anfang besteht darin, akademische Arbeit auch als Lohnarbeit und nicht (ausschließlich) als Berufung oder Privileg zu begreifen. Nur dadurch wird es möglich, Distanz zur neoliberal (selbst-)ausbeutenden 
Genie-Romantik aufzubauen (vgl. Müller/Speck 2016). Das Streben nach einer solchen Selbstdarstellung führt nicht nur zur Überforderung von Akademiker_innen, sondern häufig auch zu einer Verunmöglichung von kreativen und kollektiven Arbeits- und Lernweisen, nach denen wir, als in akademischen Kontexten Lehrende, Lernende und Arbeitende, suchen. So ließe sich mit den Worten von Stefano Harney und Fred Moten sagen:

„But this is the position of professionalization, and it is this position that confronts that student, however rare, who comes to public administration with a passion. Any attempt at passion, at stepping out of this scepticism of the known into an inadequate confrontation with what exceeds it and oneself, must be suppressed by this professionalization." (2013: 35)

Dies bedeutet, sich daran erinnern zu lassen, wie es war, Student_in zu sein und im besten Fall auch an die Zeit außerhalb der Akademie und vor der Herausbildung eines professionalisierten und akademisierten Selbst. Dies bedeutet auch, die Universität - und alle daran anschließenden Formate wie Lehre, Tagungen und Workshops - als Ort der gesellschaftlichen Reproduktion von Herrschaft kollektiv zu untergraben und uns immer wieder selbst daran zu erinnern, dass wir nicht im Leistungsparadigma aufgehen müssen und sollten. Eine solche Praxis gelingt am besten, wenn wir solidarisch und kollektiv innerhalb der Akademie zusammenarbeiten und uns Räume erspielen und erkämpfen, in welchen solche Versuche möglich sind. So helfen wir einander dabei, das notwendige Scheitern an den Leistungsansprüchen und den eigenen Idealen zugleich besser zu ertragen.

„It is rather that to be a critical [and feminist] academic in the university is to be against the university."[6]

Es war erst am letzten Tag des Treffens beim Mittagessen, dass wir uns als Orga-Gruppe zu einer Besprechung zusammensetzten. Die Zeit während des Treffens war verflogen, es war fast in Vergessenheit geraten, abzusprechen, wie all die angefallenen Aufgaben bewältigt wurden. Wir ließen uns mit einem individuell schlechten Gewissen auf ein Sofa fallen. In diesem Moment, in dem wir den anderen aus der Orga-Gruppe gegenübersaßen, bemerkten wir, wie sehr wir die Tage ausgekostet und wie wenig wir uns um organisatorische Aufgaben gekümmert hatten. Es war ein spontanes Gefühl des Scheiterns an der Erfüllung der eigenen Zuständigkeiten, einhergehend mit der Befürchtung, dass die Anderen aus der Gruppe deswegen zu viele Aufgaben zu bewältigen gehabt hätten. Doch die Überraschung war: Wir aus dem Orga-Team hatten alle ein ganz ähnliches Gefühl, nämlich einerseits zu wenig unter Kontrolle gehabt zu haben und andererseits das Treffen sehr genossen zu haben, sodass trotzdem - oder gerade deswegen - alles wie am Schnürchen gelaufen war.[7]

\section{Endnoten}

[1] Feministische Geograph_innentreffen fanden zwischen 1989 und 2004 als studentische Treffen ein- bis zweimal jährlich an wechselnden Orten im deutschsprachigen Raum statt und wurden 2015, zunächst in Seminarform, wiederbelebt. 2016 fand ein studentisch autonom organisiertes Treffen in Frankfurt am Main statt. 
[2] Die Autorinnen sind oder waren an unterschiedlichen deutschen Universitäten oder Forschungseinrichtungen angestellt.

[3] Hiermit sind alle Teilnehmenden des Vernetzungstreffens gemeint. Ohne die Ermutigung von Anne Vogelpohl hätten wir diesen Artikel nicht angefangen und ihn ohne die gemeinsame Nachbereitung des Treffens mit Linda Pasch und Ronja Lührs nicht in dieser Form zu Ende gebracht. Danke!

[4] Bei dieser Form des BarCamps gab es zunächst ein offenes Brainstorming, um Themen, für die die Teilnehmer_innen ein Austauschinteresse mitbrachten, zu sammeln. In einem zweiten Schritt haben sich Kleingruppen zu den unterschiedlichen Themenkomplexen gebildet und darüber verständigt, wie sie sich mit dem jeweiligen Thema auseinandersetzen wollen. Hierbei waren unterschiedlichste Formate vorstellbar: Textdiskussionen, Begehung des Feldes oder Erfahrungsaustausch.

[5] Duggan/Muñoz (2009: 280).

[6] Moten/Harney (2013: 31, Anmerk. d. A.).

[7] Wir werden diese Gespräche - zusätzlich zu vielen neuen Themen - beim nächsten Vernetzungstreffen „Feministische Geographien“voraussichtlich in Bonn 2018 vertiefen. Das Treffen ist offen und niedrigschwellig, Hinweise und News hierzu finden sich unter: http://ak-geographie-geschlecht.org/category/konferenzen/.

\section{Autor_innen}

Wir leben in Hamburg, Berlin und Münster, und uns eint eine akademische und politische Begeisterung für urbane Prozesse und Feminismus. Zur Zeit des Treffens haben wir alle an deutschen Universitäten oder Forschungseinrichtungen gearbeitet. Eine Autorin hat diesem Beruf nun (erst mal) den Rücken zugekehrt, auch aufgrund der Arbeitsbedingungen in der Wissenschaft. Wir sind unterschiedlich in Lehre, Antragsstellungen, Forschungsprojekte und Promotionen involviert. Nebenher versuchen wir Zeit zur Erprobung kollektiver, solidarischer und feministischer Wissensproduktion zu finden.

nina.fraeser@hcu-hamburg.de s.klosterkamp@uni-muenster.de kuehn.juliane@googlemail.com eva.kuschinski@hcu-hamburg.de theresa.martens@isinova.org

\section{Literatur}

Bürk, Thomas (2016): „Es kann nicht nur darum gehen, dass wir David Harvey unterrichten“. Interview mit Thomas Bürk zu Arbeitsbedingungen und Möglichkeiten kritischer Lehre an der neoliberalen Universität. In: $\mathrm{s} u \mathrm{~b} \backslash \mathrm{u} \mathrm{r}$ b a n. zeitschrift für kritische stadtforschung 4 (2/3), 233-240.

Dölling, Irene (2013): 30 Jahre feministische Studien: Wie mit dem feministischen Erbe umgehen? In: feministische studien 31/1, 29-34.

Dück, Julia / Fried, Barbara (2015): Caring for strategy. http://www.zeitschrift-luxemburg. de/caring-for-strategy/ (letzter Zugriff am 24.08.2017).

Duggan, Lisa / Muñoz, José (2009): Hope and hopelessness: a dialogue. In: Women and Performance: a journal of feminist theory 19/2, 257-283.

Gutiérrez-Rodríguez, Encarnación / Ha, Kien Nghi / Hutta, Jan / Ngubia Kessé, Emily / Laufenberg, Mike / Schmitt, Lars (2016): Rassismus, Klassenverhältnisse und Geschlecht an deutschen Hochschulen. Ein runder Tisch, der aneckt. In: s u b $\backslash$ u r b a n. zeitschrift für kritische stadtforschung 4 (2/3), 161-190.

Harney, Stefano / Moten, Fred (2013): The Undercommons: Fugitive Planning \& Black Study. Wivenhoe u. a: Minor Compositions.

Kratzsch, Claudia / Maruschke, Robert (2016): Basisorganisierung verändert die politische Landschaft. In: s u b $\backslash$ u r b a n 4 (2/3), 103-112. 
Marquardt, Nadine / Schreiber, Verena (2015): Mothering urban space, governing migrant women: the construction of intersectional positions in area-based interventions in Berlin. In: Urban Geography 36/1, 44-63.

Maier, Anja (2011): Die Weiber denken, sie wären besser. Kaffeehauschefin über MacchiatoMütter. http://www.taz.de/!5110280/ (letzter Zugriff am 24.08.2017).

Maruschke, Robert (2014): Community Organizing. Zwischen Revolution und Herrschaftssicherung. Eine kritische Einführung. Münster: edition assemblage.

Mountz, Alison / Bonds, Anne / Mansfield, Becky / Loyd, Jenna / Hyndman, Jennifer/ Walton-Roberts, Margaret / Basu, Ranu / Whitson, Risa / Hawkins, Roberta / Hamilton, Trina / Curran, Winifred (2015): For slow scholarship: a feminist politics of resistance through collective action in the neoliberal university. In: ACME: An International Journal for Critical Geographies 14/4, 1236-1259

Müller, Annekathrin / Speck, Sarah (2016): And the winner is.... The male academy oder: Die ungleichen Auswirkungen universitärer Prekarität. In: s u b $\backslash$ u r b a n. zeitschrift für kritische stadtforschung 4 (2/3), 203-212.

Mürlebach, Mara / Fraeser, Nina / Golke, Jürgen / Isselstein, Eva / Klosterkamp, Sarah / Kordes, Jan / Lorenzen, Kristina / Vogelpohl, Anne (2017): Von Reflexivität, Unplanbarkeit und Kollektivität im Forschungsprozess: Lasst uns die Unzulänglichkeiten der eigenen Arbeit solidarisch besprechen! In: Feministische Geo-RundMail 72, August 2017, 22-24.

Schuster, Nina (2016): Neue Horizonte feministischer Wissensproduktion. In: s u b $\backslash$ u r b a n. zeitschrift für kritische stadtforschung 4 (2/3), 191-202.

Speck, Sarah (o. J.): Latente Beharrlichkeiten. http://www.logbuch-suhrkamp.de/sarahspeck/latente-beharrlichkeiten/ (letzter Zugriff am 24.08.2017). 
